\title{
VARIA
}

\section{UNA APORTACIÓN A LA OBRA DE JEAN MIETTE}

El conocimiento de la pintura en esmalte de Limoges en los siglos XVI y XVII sigue presentando algunos enigmas especialmente a la hora de deslindar las personalidades de determinados artistas. Las condiciones en que desarrollaron su trabajo no facilitan el estudio del estilo particular de cada esmaltador y la evolución de su propia manera de hacer. Los datos biográficos sobre artistas individuales son raros. Los archivos de Limoges han proporcionado fechas de matrimonio, bautismos, etc. dentro de las familias, información sobre algunos viajes realizados por los artistas o los años en que fueron elegidos cónsules pero son muy limitados los datos sobre su actividad profesional. Por otra parte, la producción de esmaltes en Limoges conservo durante mucho tiempo un carácter artesanal y, muy frecuentemente, las obras fueron creaciones colectivas de taller siendo numerosas e importantes las dinastías de esmaltadores.

En este sentido la personalidad de Jean Miette o Myette continua siendo bastante confusa si bien con algunos interrogantes se le van atribuyendo piezas que pueden responder al todavía escaso conocimiento de sus propios caracteres estilísticos y técnicos. El descubrimiento llevado a cabo por H. Tait de las iniciales I. M. en el reverso de una taza decorada con una representación del Banquete de los Dioses, en el British Museum, Londres ( Inv. N1 $55,6-2,19)$ identificadas con este Maestro abrió el camino para posteriores atribuciones ${ }^{1}$. En parte la dificultad para definir la figura de Jean Miette, lo mismo que ha ocurrido en el caso de otros esmaltadores fue la ingente producción de talleres como los de Penicaud o Pierre Reymond a los que se iban asignando muchas obras que, aunque a veces difieren del estilo del maestro, pueden encajar en el amplio concepto y variedad de un taller. De hecho piezas relacionadas con Jean II o Jean III Penicaud o con Pierre Reymond se consideran ahora como de Miette. Es el caso, entre otros, de un plato con el tema del sacrificio de Isaac en la Colección de Los Angeles County Museum of Art que, en su momento figuró como del taller de Pierre Reymond ${ }^{2}$ o el medallón del emperador romano Vitellius que venía atribuyéndose a Jean II Penicaud ${ }^{3}$.

Así pues el monogramista I. M., activo en la segunda mitad del siglo XVI, puede tratar de identificarse con el esmaltador Jean Miette nombrado en los documentos del hospital de Li-

1 Tait, H. The Waddesdon Bequest: The Legacy of Baron Ferdinand Rothschild to the British Museum. London: Trustees of the British Museum, 1981

${ }^{2}$ Caroselli, S. L. The Painted Enamels of Limoges. A Catalogue of the Collection of the Los Angeles County Museum of Art, 1993, 150-155.

${ }^{3}$ Verdier, Ph. The Walters Art Gallery. Catalogue of the Painted Enamels of the Renaissance. Baltimore 1967, $195-196$. 
moges en 1563 y $1565^{4}$. Figura entre los artistas que Leonardo Limosin llevó en 1564 a Burdeos junto con Jean III Penicaud y otros miembros de la familia Limosin. En esta ciudad francesa se realizaron decoraciones para la entrada ceremonial de Carlos IX y su madre Catalina de Medicis. Con tal motivo el Alcalde y los Jurados habían llamado al célebre esmaltador y a sus discípulos para dessigner et paindre les (ornements et emblesmes que les dicts sieurs Maire et $J u$ ) rats ont convenu dresser et apposer pour l'entrée du Roy Charles IX. El Maestro llegó con François et autre François Lymosin, Jean Penicaud et Jean Miette, Peintres de Limoges ${ }^{5}$. Se puede suponer que Miette fuera aprendiz en el taller de Limosin y probablemente su colaborador. Aunque los esmaltadores de Limoges no realizaron joyas esmaltadas durante su estancia en Burdeos seguramente se llevaron encargos.

Este maestro es reconocible por su preferencia por la decoración de roleos y volutas muy ligeros que cuando no son dorados parecen plateados y están asociados en los bordes con la inserción de medallones ovales que recuerdan camafeos y entalles antiguos. En relación con esto hay que anotar que bordes con medallones como camafeos pueden haber sido pintados sobre aceites o dibujados en bistre. El estilo de las figuras de cuerpos gruesos y alargados demuestra la influencia de Leonard Limosin y el trabajo manierista italiano en la corte francesa. Hablando en lenguaje pictórico diríamos que hay un predominio de las formas casi etéreas que, a veces, parecen diluirse, mientras la delineación de los contornos se muestra imprecisa. El tema principal viene a primer plano envuelto en una excelente ambientación donde se marcan los planos en profundidad con una gradación de tonalidades en tanto que los perfiles se difuminan.

A Jean Miette podemos atribuir dadas sus características un plato con el tema del Laocoonte perteneciente a la Colección Thyssen Bornemisza (Ref. E. 13, diám. 40,5 cm., adquirido antes de 1938). Tradicionalmente viene relacionandose con el taller de Pierre Reymond pero sin duda está más próximo y tiene más puntos en común con este pequeño grupo de objetos identificados con Jean Miette. El borde está constituido por una banda en blanco opaco con líneas rojas. En el anverso el caveto muestra una orla en fondo azul en la que cuatro máscaras alternan con cuatro medallones ovales. Por los ojos de las máscaras pasan unos tallos que se van enroscando en curva y contracurva para finalizar en unas cabezas de dragones de bocas abiertas y largas lenguas rojas. Las máscaras serán motivos repetidos al igual que en la arquitectura y la escultura del siglo XVI. Al parecer se trata de una trasposición de las máscaras de oro precolombinas lo que supone la inclusión de motivos exóticos en diversas manifestaciones artísticas ${ }^{6}$.

Los cuatro medallones ovales, imitando camafeos en relieve, incluyen sobre fondo negro representaciones de figuras cabalgando sobre animales marinos. En el del eje superior se muestra una figura desnuda con una banderola en su mano movida por el viento, sobre un buey marino. Siguiendo hacia la derecha observamos otra figura, también con banderola en su mano, sobre una especie de delfín con escamas; la siguiente es una figura que cabalga sobre un tipo de toro cuyos cuartos traseros se convierten en una gran cola. Su esquema compositivo y su forma de representación recuerda las habituales para el Rapto de Europa. Finalmente una figura con los brazos levantados sobre un delfín plasma la repetida escena del niño desnudo que viaja por encima de las olas sobre el mamífero marino. Este animal sagrado jugó un papel

\footnotetext{
${ }^{4}$ Guibert, L. «Catalogue des artistes limousins», Bulletin de la Societé archeologique et historique du Limousin, 58, 1908,193, n. $^{\circ} 842$

${ }^{5}$ Braquehaye, M. CH. «Les Peintres de l'Hôtel de Ville de Bordeaux et des entrées royales despuis 1525», Reunion des Societés des Beaux Arts des Departements, Paris 21 session, 1897, 846-848.

${ }^{6}$ Collon-Gevaert, S. «L'Art precolombien et le palais des princes- évéques à Liege». Bulletin de la Societé d'Art et d'Histoire du Diocese de Liege, XLI, 1959, 73-95.
} 

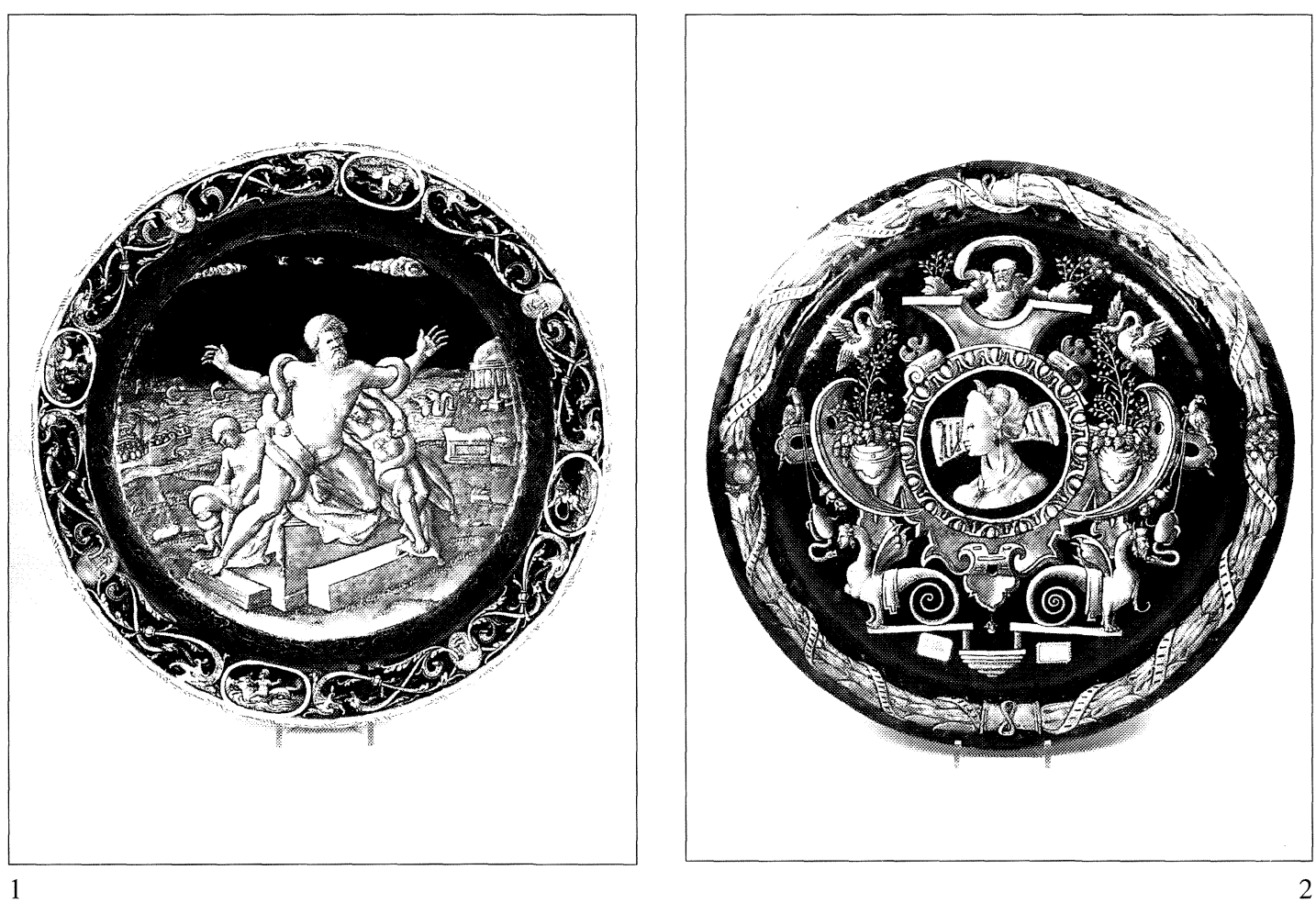

3

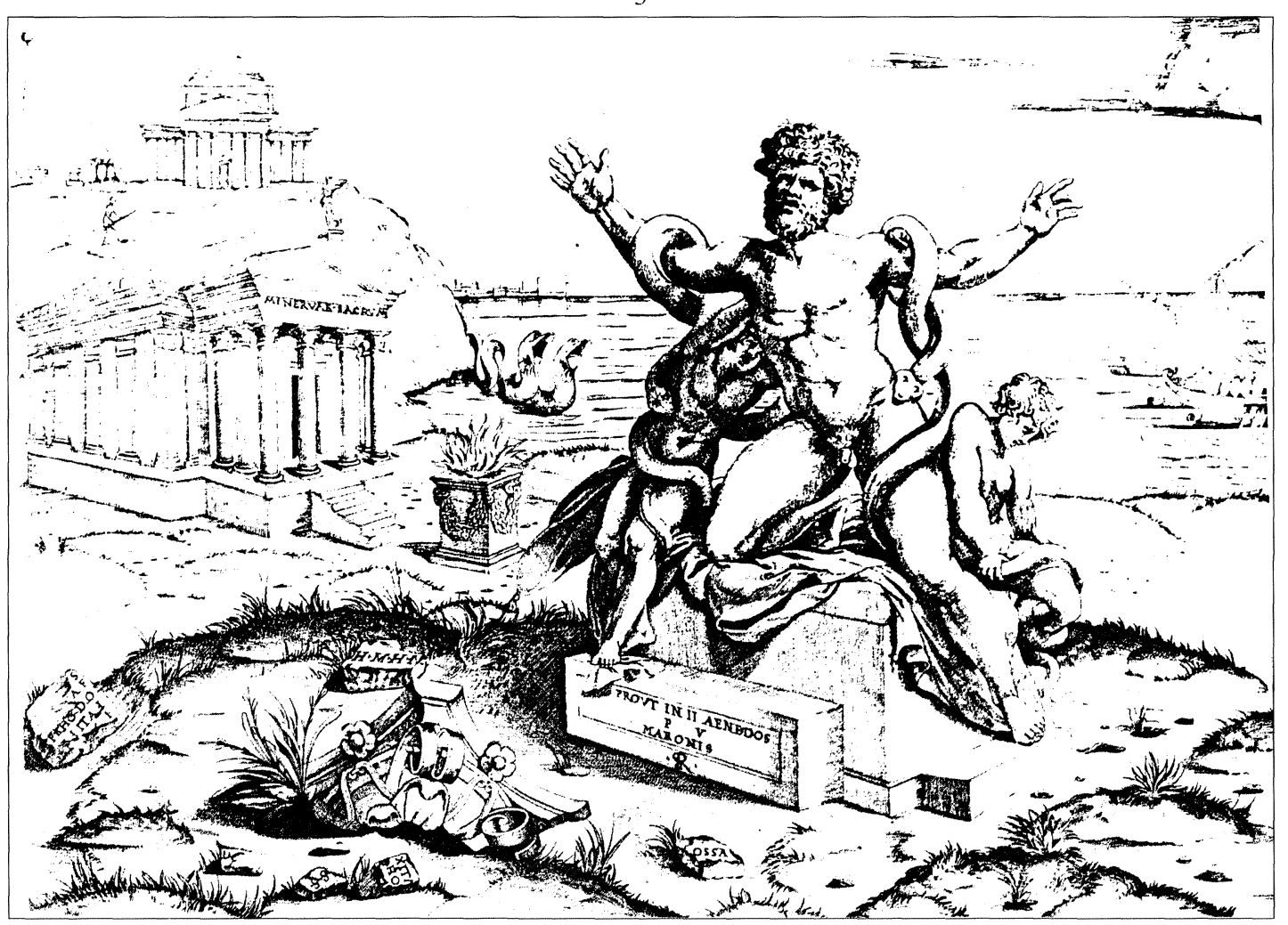

Figs. 1-2. Plato de esmalte sobre cobre con el Laoconte.Madrid, Museo Thyssen Bornemisza.

Fig. 3. M. Raimondi: Laoconte. 
importante en los ritos funerarios en el mundo griego donde aparece como psicopompo siendo asimismo símbolo de prudencia, divinización y sabiduría. El tema parece identificable con el motivo extraído de la recopilación anónima conocida como Gesta Romanorum, fuente fundamental de inspiración para los artistas del Renacimiento ${ }^{7}$. Un segundo encuadramiento, en este caso con roleos de trazo muy fino, marca el paso a la cavidad del plato.

La representación de Laocoonte y sus hijos atacados por las serpientes constituye el tema central. Sacerdote de Apolo y Poseidón había alertado a los troyanos contra el caballo abandonado por los griegos delante de su ciudad. Mientras preparaba un sacrificio dos serpientes salen del mar y enroscan al sacerdote y a sus hijos. Apolo le hacía así expiar un sacrilegio que había cometido anteriormente contra él. Los troyanos vieron en esta muerte ordenada por los dioses el signo de que Laoconte se había equivocado en su advertencia e introdujeron el caballo en la ciudad. La escena se desarrolla en un ambiente de agitado mar al fondo con las cabezas de algunos monstruos marinos y el ara del sacrificio donde la nota de color la pone el fuego. El momento elegido es el de máxima tensión. Los cuerpos de acusada musculatura transmiten el sentimiento de lucha al mismo tiempo que de impotencia y angustia. En primer plano la imponente figura del sacerdote, de musculosa anatomía, levanta sus brazos hacia el cielo. A ambos lados sus hijos tratan de liberarse de las serpientes que les atenazan. El fondo es de esmalte azul oscuro. El esmalte blanco opaco en que están hechos los dibujos se adelgaza creando un profundo gris-azulado. El color carne se logra mediante un baño de esmalte rojo en fundente claro sobre áreas de blanco espeso. El rojo se utiliza para el fuego sobre el ara, las lenguas de los dragones, la decoración del borde, las piezas de fruta sobre la orla del reverso y algún otro detalle.

La historia de la guerra de Troya había sido transmitida desde la Antigüedad por Homero y Virgilio principalmente, aunque de forma diferente. Mientras Virgilio relata con detalle el episodio de Laocoonte, Homero guarda silencio en la Iliada. Sin embargo ahora la historia del sacerdote y sus hijos se ve magníficamente ilustrada por el hallazgo en Roma en enero de 1506 de las esculturas en mármol. El grupo descubierto en las termas de Tito sobre el Esquilino fue ofrecido al papa Julio II que lo instaló en el Belvedere. Identificado con el mencionado por Plinio el Viejo en su Historia Natural (XXXVI, 37), despertó el entusiasmo de artistas, poetas y humanistas. Una copia de bronce fue encargada por Francisco I en 1540 a Primaticio para la Galeria de los Ciervos en Fontainebleau ${ }^{8}$.

Este grupo se convirtió en un tema de estudio para los artistas del Renacimiento y, entre otras cosas, numerosos grabados ilustran el apasionamiento suscitado por la escultura. El más antiguo conocido es obra de un discípulo de Raimondi, Marco Dente llamado Marco de Ravenna, muerto en 1527. Firmó dos grabados con la representación del Laocoonte, ambos utilizados como modelos por los esmaltadores de Limoges. Uno de ellos representa el grupo antiguo antes de la restauración. El otro, que se conserva en Viena, es el empleado en este caso, en una composición invertida, tal como puede apreciarse ${ }^{9}$.

El empleo de grabados como fuente de inspiración entre los esmaltadores de Limoges estuvo muy difundido. Predominan en un primer momento los de temática religiosa derivados de composiciones de los Paises Bajos y Borgoña. Posteriormente, más o menos a partir de 1530, en Francia circularon diversas colecciones de grabados, seguidas por libros franceses ilustrados, especialmente novelas cortesanas, biblias y antologías mitológicas. Las estampas de Raimondi a veces conocidas a través de copias inspiraron a la mayoría de los maestros desde Leo-

\footnotetext{
${ }^{7}$ Müntz, E. Histoire de l'art pendant la Renaissance, I, Italie. Les Primitifs. Paris 1889, 245.

8 Andreae, B. Laokoon und die Kunst von Pergamon, die Hybris des Giganten, Frankfort, 1991, 6, fig. 6.

${ }^{9}$ Bartsch, The Works of Marcantonio Raimondi and his School, New York, 1978, vol. 26, n. ${ }^{\circ} 243,240$ y vol. 27, n. ${ }^{\circ} 353,50$.
} 
nard Limosin a Pierre Reymond pero los artistas limosines pronto se sintieron atraídos por la libertad y novedad de inspiración introducida desde Italia por los pintores de la Escuela de Fontainebleau. En la segunda mitad del siglo, después de la muerte de Francisco I, grabadores de libros ilustrados como Etienne Delaune y Bernard Salomon serán los modelos favoritos. Copiaron tanto estampas completas como se limitaron a reproducir algunas partes que les gustaban más y, aunque no parece exacto, algunos autores opinan que no fueron capaces de imaginar composiciones considerandoles artesanos desprovistos de poder creador ${ }^{10}$.

Entre las piezas más conocidas con este mismo tema del Laocoonte hay que recordar la gran placa de Pierre Courteys de hacia 1560, en el Musée Municipal de l'Eveché en Limoges (inv. 88453) ${ }^{11}$, basada en el primer grabado de Marco Dente . Este mismo grabado sirve a Pierre Penicaud en fecha similar para la ejecución de una placa oval en grisalla en el Museo Civico de Turín (inv. SM13). En 1539 Leonard Limosin reprodujo en grisalla, sobre una placa cóncava en el fondo de una copa en el Victoria \& Albert Museum ( inv. 544-1853), una escena comparable, inspirada en el otro grabado de Marco Dente según Rafael ${ }^{12}$. Esta segunda versión ha sido igualmente utilizada, invertida, por M.D. Pape y P. Courteys en 1552 para otras copas en colecciones privadas y asimismo se emplea también en el plato de la Colección Thyssen Bornemisza.

En el reverso del plato la orla está formada por una corona de laurel anudada en la parte superior e inferior y rodeada por un encintado. En el centro, un disco bordeado por la consabida decoración de ovas y flechas encuadra un busto femenino de perfil, con una especie de cartela con barras rojas al fondo. Su representación de porte majestuoso y expresión serena y grave, su cabeza tocada con una diadema y un velo recuerda algunas representaciones de Juno. El perfil de nariz recta como prolongación de la frente, boca de labios finos y barbilla redondeada se aproxima a la placa con la representación del carro de Juno de Leonard Limosin en la Colección Rothschild ${ }^{13}$. A los lados dos medias lunas cobijan sendos búcaros con flores, frutos y dos aves de larguísimos cuellos en la parte superior. En el eje, por encima de la cartela, la figura de un anciano con coraza y una banda que simula una cúpula, quiere recordar a Caelus. Completan la decoración dos esfinges en la parte baja apoyadas en una especie de plataformas. El fondo es azul salpicado de puntitos dorados.

El repertorio decorativo a base de cartuchos, máscaras, cueros recortados, guirnaldas de flores y frutos, hojas, etc. adornan las piezas al modo de los estucos de la Escuela de Fontainebleau cuyas composiciones se convertirán en punto de referencia obligado para los esmaltadores de Limoges. Generalmente se enrollan alrededor del tema principal y emblemas y atributos se disponen siguiendo una composición en eje de simetría.

El hecho de que esta pieza no lleve marcas ni iniciales hace que tengamos que hablar de atribución y no de identificación. Sin embargo, las concomitancias especialmente con dos platos de la Walters Art Gallery con escenas del Antiguo Testamento que muestran la Creación y el sacrificio de Caín y Abel, y uno con perfil del emperador Vitellius que Verdier atribuye al Maestro I.M. (Jean Miette?) ${ }^{14}$ son más que evidentes por lo que podría pasar a formar parte del grupo de obras relacionadas con este maestro.

M. ${ }^{a}$ LuISA MARTín AnSÓN

U.A.M.

\footnotetext{
${ }^{10}$ Marquet de Vasselot, J.J. Les émaux limousins de la fin du XVe siècle et de la premiere partie du XVIe., Paris, $1921,6$.

11 Notin, V. Notice de presentation au Musée Municipal de l'Evêché,Ville de Limoges, 20 fevrier 1989. El mismo texto y su traducción al castellano está publicado en L'esmalt, set. 1991, 8-10.

Exposition, Tresors d'email, Limoges. Musée Municipal de l'Eveché, 1992, 158-159

12 Baratte, S. Leonard Limosin au Musée du Louvre, Paris 1993, 70

13 Marchaix, M. «Limoges Enamels», The James A. de Rothschild Collection ad Waddesdon Manor. Glass and Enamels, Friburgo 1977, 332.

14 Verdier, Ph. Op. cit. 1967, 191-196.
} 